\title{
Imaging spectrum of pulmonary infections in renal transplant patients
}

\author{
Zohra Ahmad, Soumita Bagchi¹, Priyanka Naranje², S K Agarwal1, Chandan J Das² \\ Department of Radiology, Gauhati Medical College, Assam, Departments of ${ }^{1}$ Nephrology and ${ }^{2}$ Radiology, AlIMS, \\ Ansari Nagar, New Delhi, India \\ Correspondence: Dr. Chandan J Das, Department of Radiology, All India Institute of Medical Sciences, Ansari Nagar, New Delhi - 110029 , \\ India. E-mail: docchandan17@gmail.com
}

\begin{abstract}
In the post renal transplant setting, pulmonary infections comprise an important set of complications. Microbiological diagnosis although specific is often delayed and insensitive. Radiography is the most common and first imaging test for which patient is referred, however it is relatively insensitive. HRCT is a very useful imaging tool in the scenario where radiography is negative or inconclusive and high clinical suspicion for infection is present. HRCT features vary among the various pathogens and also depend on the level of immunocompromise. Certain HRCT findings are characteristic for specific pathogens and may help narrow diagnosis. In this review article, we will summarize the imaging findings of various pulmonary infections encountered in post renal transplant patients.
\end{abstract}

Key words: High resolution computed tomography, immunocompromised, peritransplant, pneumonia, renal transplant

\section{Introduction}

In the immunocompromised transplant recipient, several pulmonary complications are prevalent of which three-fourth are infections ${ }^{[1]}$ Early diagnosis and treatment for pulmonary infections is of paramount importance due to its associated high morbidity and mortality. One of the important factors affecting the spectrum of infections is timing since renal transplant. Early diagnosis of infection and narrowing down the possible differential diagnosis helps significantly in deciding appropriate treatment. Palin radiograph of chest, though is the first investigation for clinical work up, is neither sensitive nor specific for specific etiology of pulmonary infections. In majority of patients, additional imaging modalities like $\mathrm{CT}$ chest and additional investigations like BAL and percutaneous lung biopsy are often required for reaching to a specific diagnosis. In this

\begin{tabular}{|c|c|}
\hline \multicolumn{2}{|c|}{ Access this article online } \\
\hline Quick Response Code: & \\
\hline \multirow{2}{*}{ 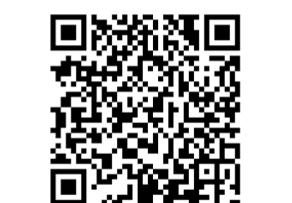 } & $\begin{array}{l}\text { Website: } \\
\text { www.ijri.org }\end{array}$ \\
\hline & $\begin{array}{l}\text { DOI: } \\
\text { 10.4103/ijri.IJRI_357_19 }\end{array}$ \\
\hline
\end{tabular}

respect, HRCT is an important tool with a high negative predictive value.

\section{Imaging modalities}

- Radiography is a useful modality for initial screening and follow up, where a definite abnormality is detected. In a neutropenic patient, due to decreased inflammatory response, often radiographic findings are minimal, and this modality alone may not suffice. Obvious advantages include low radiation, frequent repeatability, and bedside/ICU availability. Radiography is useful in detecting pleural effusion, large consolidation, widespread lung abnormality and large sized nodules. Flip side of this modality is low sensitivity and the inability to detect focal ground glass

This is an open access journal, and articles are distributed under the terms of the Creative Commons Attribution-NonCommercial-ShareAlike 4.0 License, which allows others to remix, tweak, and build upon the work non-commercially, as long as appropriate credit is given and the new creations are licensed under the identical terms.

For reprints contact: WKHLRPMedknow_reprints@wolterskluwer.com

Cite this article as: Ahmad Z, Bagchi S, Naranje P, Agarwal SK, Das CJ. Imaging spectrum of pulmonary infections in renal transplant patients. Indian J Radiol Imaging 2020;30:273-9.

Received: 18-Sep-2019 Accepted: 06-Jul-2020

Revised: 24-May-2020 Published: $15-$ Oct-2020 
abnormality, small localized nodules, and abnormality in hidden areas. One of the major advantages of radiography is to give the initial impression of etiology of infection by pattern of lung involvement so that initial empirical antibacterial can be started pending definitive diagnosis

- High-resolution computed tomography in immunosuppressed patients is mostly required to assess more details of the extent of pulmonary involvement and pattern of involvement. Further, it becomes mandatory in a patient with pulmonary symptoms with normal chest radiograph. It may be advisable to consider thin section CT in all patients with neutropenia and normal chest radiographs. In a study of 20 patients, Gulati et al. found that HRCT revealed additional findings as compared to radiography in 11 patients. Seventeen of the 20 patients had a final diagnosis of infection of which 11 were diagnosed by HRCT and the final diagnosis coincided with HRCT diagnosis in all except one patient. In some cases, the imaging patterns are specific or highly suggestive of a particular infection like nodule with halo in invasive aspergillosis, bilateral ground glass, or reticular opacity in cytomegalovirus (CMV)/ Pneumocystis jiroveci pneumonia (PCP) and bulging fissure sign in Klebsiella infection. This helps us in narrowing the differential diagnosis and guiding the specific treatment

- Ultrasound Its use is limited to detecting pleural effusion on bedside and guiding interventions like pleural fluid aspiration or guided pulmonary biopsy.

\section{Timeline of infections}

Pulmonary infections after renal transplantation are most common in the first 6 months with a peak at about 3 months. During the first month, infections are similar to patients undergoing other thoracic and abdominal surgeries. During this period, the common infections are pneumonia due to gram negative bacterial infections and septic emboli due to intravenous lines. Between months 1 and 6, common infections are viral (CMV, EBV, and HSV), fungal (aspergillus, PCP), and tubercular infections. Viral infections may also predispose to fungal infections [Table 1].

\section{Common infections in peri-transplant period}

In recent studies by Gandhi et al. (2018) and Japerumal et al. (2016), bacterial infection followed by tuberculosis and fungal infections were found to be the most common

Table 1: Timeline of infections in renal transplant patients

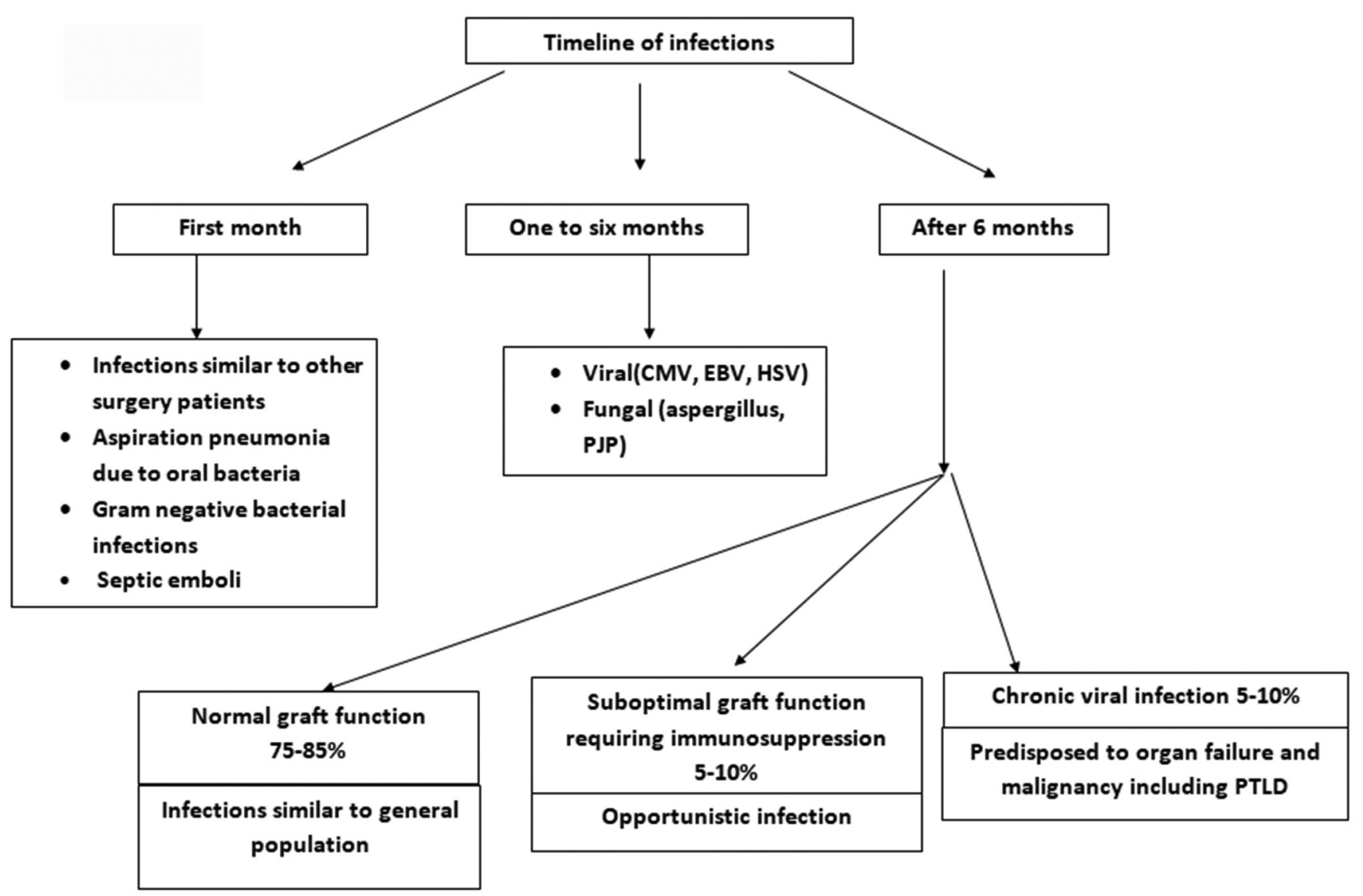




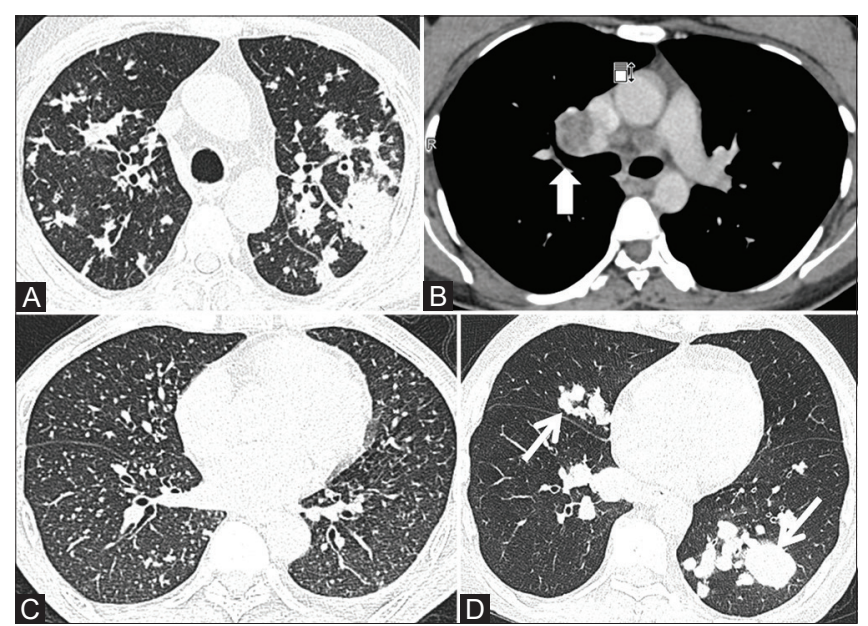

Figure 1 (A-D): (A-D) Bacterial infections (A) Secondary tuberculosis pattern. Axial HRCT shows multiple ill-defined nodules and patchy consolidation with upper lobe predominance in a patient of multidrug-resistant tuberculosis. (B) Mediastinal lymphadenopathy in tuberculosis. Axial NCCT shows necrotic mediastinal lymphadenopathy in right paratracheal location (arrow). (C) Miliary tuberculosis. Axial HRCT shows numerous small, uniform 2-3mm nodules in both lungs without any apicobasal gradient. (D) Nocardia. Patient presented with fever. Axial HRCT shows multiple well-defined nodules and masses (arrows) without air bronchograms and not showing any apicobasal gradient. CT-guided biopsy from the lower lobe mass confirmed the diagnosis

infections in renal transplant recipients. Another study by Affara et al. (2015), bacterial followed by mixed bacterial, tuberculosis, and CMV infections were found to be most common in the first year after renal transplant. Wang et al., found mixed infections, followed by virus and bacterial as the most common etiology. ${ }^{[2]}$ Another study from India found tuberculosis to be most common infection in the group of patients they studied. ${ }^{[3]}$

\section{Bacterial}

Imaging findings in bacterial infections in these patients are not much different from the general population. However, there is a propensity to rapid progression and complications like abscess and bacteremia, if patients are not treated promptly in time.

\section{Mycobacterial infections}

Mycobacterium tuberculosis comprises $10-15 \%$ of pulmonary involvement in kidney transplant patients in endemic areas like India, though it is much less in Western countries ${ }^{[4]}$ A majority of tuberculosis patients will present in the first year post transplant,,$^{[5]}$ though it is still common in the later part of the posttransplant period Tuberculosis has been traditionally classified into primary and secondary. Primary tuberculosis presents as consolidations, necrotic lymph nodes, miliary nodules, and pleural effusions. Secondary tuberculosis which is due to reactivation presents as patchy consolidations with cavities more often in upper lobes in the background of fibrosis [Figure 1A].
Table 2: Tuberculosis in renal transplant patients

May show primary disease irrespective of prior exposure

Necrotic nodes, lower lung disease, and pleural effusion rather than upper lobe cavitatory disease

Tree in bud due to airway dissemination

Miliary: poor prognosis

Table 3: Gram positive bacteria in renal transplant patients

Streptococcus
Lobar pneumonia
Small effusion
Staphylococcus
Bronchopneumonia
Pleural effusion, emypema
Cavitation and pneumatoceles

Table 4: Gram negative bacteria in renal transplant patients

Pseudomonas:
Bronchopneumonia
Bilateral complex lower lobe nodules
Pleural effusion, abscess
Klebsiella
Lobar pneumonia
Pleural effusion, cavitation, and abscess
Nocardia
Nodules, consolidation, cavitation, and lymphadenopathy
Effusion, abscess
Extension to mediastinum, pericardium, and chest wall
Anaerobic bacteria
Aspiration
Pneumonia affecting posterior segment of upper lobes, superior and posterior
basal segments of lower lobes
Abscess and empyema

Renal transplant patients are immunocompromised and may present with the findings of the primary disease irrespective of prior exposure [Table 2]. ${ }^{[6]}$ Imaging findings in immunocompromised patients may include lower lung disease, necrotic adenopathy [Figure 1B], pleural effusion among other findings as compared to upper lobe cavitatory disease in immunocompetent patients. ${ }^{[7]}$ Cavitation is relatively uncommon in post renal transplant setting as compared to the general population. ${ }^{[8]}$ Tree in bud appearance which is due to airway dissemination is more common in tuberculosis as compared to other bacterial infections. Miliary nodules are an indication of severe immunocompromised state and hence may have a poor prognosis associated with higher mortality ${ }^{[9]}$ [Figure 1C]. Signs of activity in TB are consolidation, tree in bud appearance, miliary nodules and cavities, while calcified nodules, linear opacities, and bronchiectasis indicate inactivity. ${ }^{[10]}$

\section{Gram positive bacteria}

Streptococcus, which is the most common cause of community-acquired pneumonia typically causes lobar pneumonia. It may be associated with small pleural effusions; however, abscess and empyema are uncommon. Staphylococcus causes bronchopneumonia, which may 
Table 5: Fungal infections in renal transplant patients

Aspergillus
ABPA: Central bronchiectasis with mucus plugging, tree in bud opacities
Aspergilloma: Mobile opacity in preexisting cavity
Semi-invasive: Nodules $>1 \mathrm{~cm}$, consolidation $+/$ - cavitation in upper lobes.
D/D TB
Angioinvasive: Nodule with halo, mass, wedge-shaped consolidation,
cavitation
Airway invasive: Bronchiolitis or bronchopneumonia
Candida
Multiple nodules/consolidation in lower lobes
Cryptococcus
Multiple nodules or masses, segmental or lobar consolidation in lower lobes
Mucormycosis
Large nodules, consolidations with reverse halo sign, and large areas of
peripheral GGO
Fissural and chest wall invasion
Pneumocystis jeroveci
Bilateral upper lobe and perihilar GG0 and septal thickening with subpleural
sparing
Crazy-paving, pneumatocoeles and cysts

later become confluent leading to lobar pneumonia. It may be associated with pleural effusion, emypema, cavitation, and pneumatoceles. Staphylococcus is a common cause of secondary bacterial infection in post influenza patients [Table 3]. ${ }^{[11]}$

\section{Gram negative bacteria}

Common infections in this group include Pseudomonas, Klebsiella, Acinetobacter and Enterobacter. They comprise about $69 \%$ of bacterial infections in the renal transplant setting, of which 60 percent infections are due to pseudomonas. ${ }^{[12]}$ Infection with pseudomonas is commonly due to aspiration, that is why it presents as bronchopneumonia. Imaging findings include bilateral complex lower lobe nodules, which may be associated with pleural effusions. Nodules may conglomerate to form complex masses with air bronchogram. ${ }^{[6]}$ Abscess formation may also occur in severe cases. Klebsiella infection presents as lobar pneumonia similar to pneumococcus. Klebsiella often involves upper lobes. Bulging fissure sign is fairly specific to Klebsiella. It is often associated with pleural effusion, cavitation, and abscess formation. Nocardia is a rare infection which must be kept in mind when dealing with post renal transplant pulmonary infections. Nocardia is predominantly a soil-borne pathogen, which must be inhaled; therefore, lung is often the primary site of infection. ${ }^{[13]}$ Imaging findings include nodules, consolidation, cavitation, and lymphadenopathy, which may be complicated with effusion, abscess, and extension to mediastinum, pericardium, and chest wallX [Figure 1D and Table 4]. Pneumonia due to anaerobic bacteria like bacteroides may be caused due to aspiration of oral contents. Pneumonia affecting the posterior segment of upper lobes and the superior and posterior basal segments of lower lobes may raise this possibility. It may be further complicated by abscess and empyema formation.

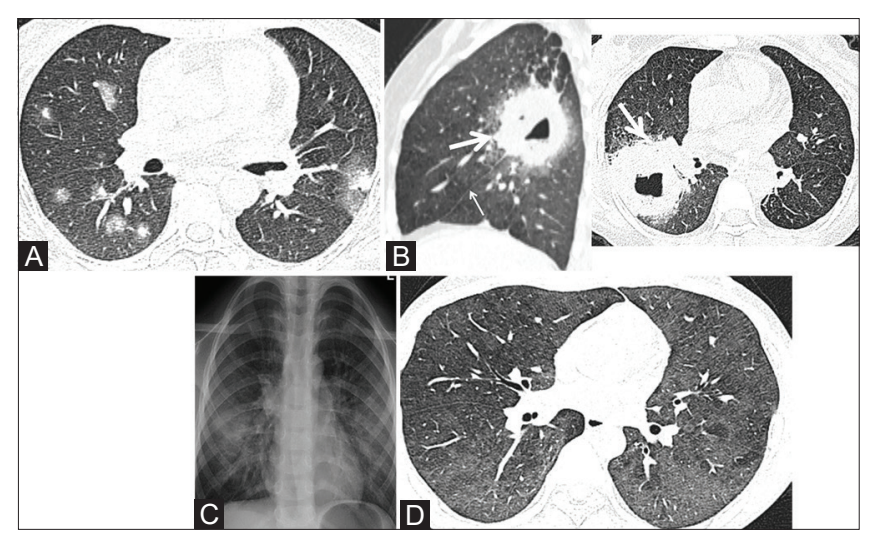

Figure 2 (A-D): (A-D) Fungal infections (A) Angioinvasive aspergillosis. Patient presented with fever and blood tinged sputum. Axial CT shows multiple nodules with surrounding ground glass opacity and consolidation. (Nodule with halo sign)(arrow). Some of the nodules revealed central cavitation (not shown). (B). Mucormycosis. Axial HRCT and sagittal reformat show a single large mass-like area of consolidation with surrounding ground glass opacity (arrow) in posterior segment of right upper lobe extending across the major fissure (thin arrow) into the superior segment of the lower lobe. Fissural invasion and perilesional GGO suggest an aggressive fungal infection. On biopsy this was proven to be mucormycosis. (C) Pneumocystis jeroveci pneumonia. Radiograph shows ill-defined air space opacity in bilateral upper and mid-zones in perihilar region. (D) Pneumocystis jeroveci pneumonia. Axial HRCT shows diffuse bilateral asymmetrical ground glass opacity with areas of interlobular septal thickening. Few scattered intrapulmonary cysts were also seen (not shown). Bronchoalveolar lavage with gomori methanamine silver staining confirmed the diagnosis

\section{Aspergillus}

The spectrum of aspergillus infection varies depending upon the severity of immunocompromised status. Aspergilloma and allergic bronchopulmonary aspergillosis (ABPA) affect patients with normal immune status. Aspergilloma is seen as a ball of fungal hyphae in a preexisting cavity. The fungal ball/mycetoma may be seen to move between prone and supine scans. It may also present as a irregular sponge-like opacity with air spaces filling a preexisting cavity. Allergic bronchopulmonary aspergillosis (ABPA) is seen as bilateral central bronchiectasis with mucus plugging and peripheral tree in bud opacities. Semi-invasive aspergillosis is seen in patients with mild immunocompromised status usually with underlying chronic lung disease like prior tuberculosis, fibrosis, or COPD. Imaging findings include nodules larger than $1 \mathrm{~cm}$, consolidation with or without cavitation in the upper lobes. ${ }^{[14]}$ Semi-invasive aspergillosis may be difficult to differentiate from active tuberculosis. Invasive aspergillosis affects patients who are immunosuppressed, and may be angioinvasive or airwayinvasive. Invasive aspergillosis has a high fatality rate approaching 75 percent. ${ }^{[15]}$ In angioinvasive aspergillosis, the typical finding of nodules with surrounding ground glass opacity representing hemorrhage is specific; however, it is uncommon [Figure 2A]. Nodule with halo sign is also described in other conditions like Wegener's granulomatosis, Kaposi sarcoma, and other infections; however, in the setting of infections not responding to 
standard antibiotics particularly in transplant setting, it is highly suggestive of angioinvasive aspergillosis. ${ }^{[16]}$ Other imaging findings can include masses and wedge-shaped consolidation. Cavitation, when present, generally occurs two weeks after the appearance of nodules. Airway invasive aspergillosis presents as bronchiolitis or bronchopneumonia. HRCT findings are peribronchial consolidation, centrilobular nodules, and tree-in-bud appearance. ${ }^{[17]}$

\section{Candida albicans}

Candida infection in a transplant setting is more commonly seen in oropharynx and esophagus. Pulmonary infection is relatively uncommon. Imaging findings include multiple nodules and focal consolidation more commonly seen in lower lobes.

\section{Cryptococcal pneumonia}

Cryptoccal infection is quite rare in a renal transplant setting. ${ }^{[18]}$ Imaging findings of cryptococcal pneumonia include nodules or masses of segmental or lobar consolidation which are often bilateral and asymmetrical. Peripheral and lower lobe distribution is more common.

\section{Mucormycosis}

The most common site of mucorinfection is rhinocerebral followed by pulmonary and disseminated infections. ${ }^{[19]}$ Common imaging findings include large nodules, consolidations with reverse halo sign (Bird nest sign) and large areas of perilesional ground glass opacity. Fissural and chest wall invasion is highly suggestive of mucormycosis [Figure 2B]. Lesions often show a peripheral predominance. Patients with severe infections may show a multifocal pneumonia pattern which is associated with poor prognosis. ${ }^{[20]}$

\section{Pneumocystic jiroveci}

Prior to the era of universal cotrimoxazole prophylaxis, P. Jiroveci pneumonia (PCP) incidence was 5-10\%, among renal transplant patients. PCP patients show severe hypoxemia, dyspnea, and cough with paucity of physical findings. Radiographs are commonly abnormal and show diffuse bilateral interstitial opacities in a perihilar distribution ${ }^{[2]}$ [Figure 2C]. Reticular and ground-glass opacities may progress to frank consolidation

\section{Table 6: Viral infections in renal transplant patients}

\section{CMV}

Diffuse bilateral GGO, interstitial opacities, consolidation

Centrilobular nodules $<10 \mathrm{~mm}$

Community respiratory viruses

Bronchiolitis and pneumonia

Ground glass opacity, centrilobular nodules, and tree in bud appearance Varicella zoster virus

Small nodules, nodule with surrounding GGO, diffuse miliary pattern, patchy GGO, and coalescent nodules

Coronavirus disease 2019 (COVID-19)

Multifocal patchy, predominantly peripheral ground glass opacities

Confluent ground glass and consolidation in later stages over 3-4 days. ${ }^{[22]}$ Hallmark appearance on CT is bilateral upper lobe and perihilar ground-glass opacities and septal thickening with subpleural sparing [Figure 2D]. Crazy-paving appearance, pneumatoceles, and cysts may also be noted, of which the latter may predispose to pneumothorax. HRCT has a high sensitivity and specificity for diagnosis of PCP; so, a normal study may help in the exclusion of the diagnosis. ${ }^{[23]}$ Imaging appearance may worsen initially during therapy due to inflammatory reaction; ${ }^{[24]}$ however, ultimately ground-glass opacities get resolved completely once therapy is continued [Table 5]. ${ }^{[25]}$

\section{CMV}

CMV is the most common virus encountered in renal transplant recipients. CMV infection ranges from subclinical viremia, a mononucleosis like CMV syndrome with fever, malaise, and neutropenia, and organ specific infection among which lung is one of the common involvement. Given that shedding virus in respiratory tract may occur without tissue invasion, the detection of CMV DNA in bronchoalveolar lavage can only suggest a presumptive diagnosis. ${ }^{[26]}$ For the above reason, it is important to correlate with clinical and radiological findings. CMV pneumonia incidence among kidney transplant patients is $2 \%{ }^{[12]}$ Moreover, CMV infection may predispose to other infections. Most common imaging findings are diffuse bilateral ground glass, interstitial opacities, consolidation, and centrilobular nodules ${ }^{[27]}$ [Figure 3A and B]. Centrilobular nodules less than $10 \mathrm{~mm}$ favor viral infection over other etiologies. ${ }^{[20]}$ The close differential is PCP infection; however, pleural effusion is more common in CMV than PCP. ${ }^{[27]}$

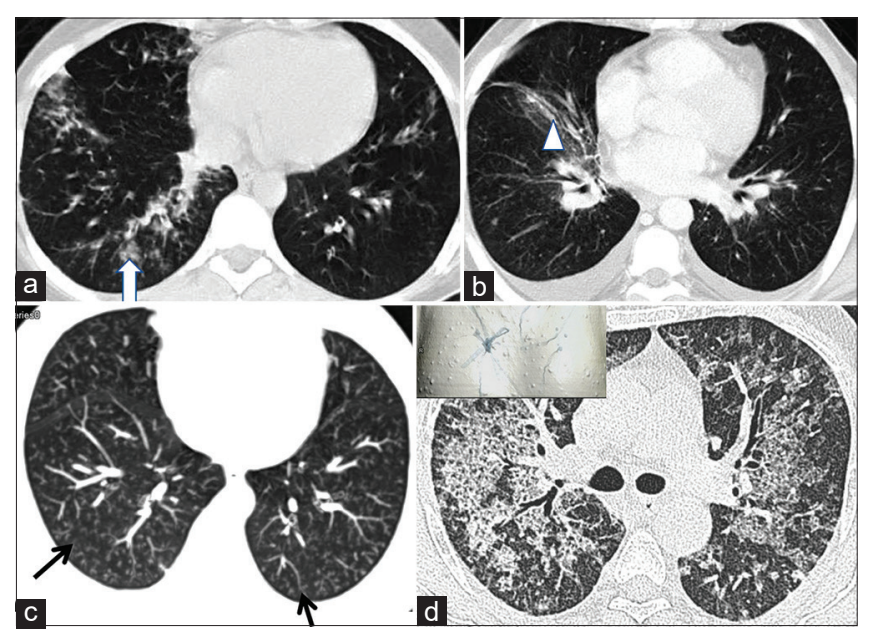

Figure 3 (A-D): (A-D) Viral Infections (A and B) CMV pneumonia. Axial HRCT shows ill-defined centrilobular nodules (arrow in $A$ ) in right lower lobe with peribronchial thickening (arrowhead in B) and bilateral pleural effusion. (C) Viral bronchiolitis. Patient presented with fever and cough. Chest radiograph was normal (not shown). Axial HRCT MIP image shows scattered centrilobular nodules (arrows) with tree-in-bud appearance in bilateral lower lobes and lingula. (D) Varicella Zoster Virus (VZV) Pneumonia. Axial HRCT shows scattered consolidation randomly distributed in both lungs. Areas of interlobular septal thickening and lobular sparing are also noted. This renal transplant patient had skin pustules typical of Varicella Zoster (inset) 


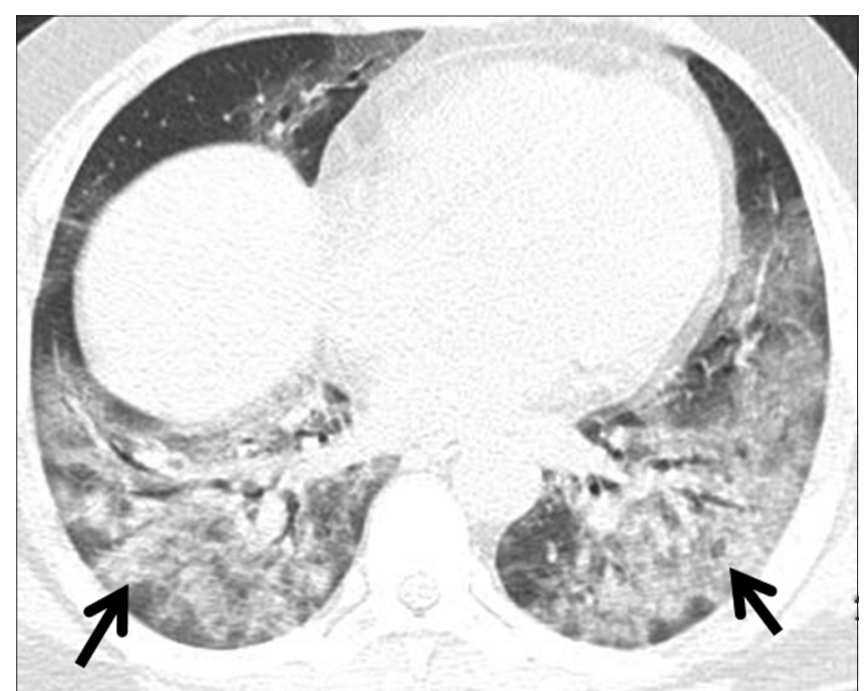

Figure 4: Coronavirus disease 2019 (COVID-19) pneumonia. Axial HRCT shows patchy ground glass opacities (arrow) in both lower lobes. The upper and middle lobes also revealed multifocal peripheral ground glass opacities (not shown)

Wang et al., found ground glass opacity as the most common radiological finding in CMV infection. On the other hand, mixed infections of CMV with other organisms had consolidation in addition to ground glass opacity. ${ }^{[2]}$ Horger $e t$ al.${ }^{[28]}$ found that the initial CT pattern of bilateral diffuse or patchy ground glass opacity followed by progressive consolidation portends poor prognosis. This pattern, in addition to the sudden change at follow up to the diffuse bilateral ground glass opacity pattern similar to diffuse alveolar damage, is a sign of unfavorable prognosis with higher fatality.

\section{Community respiratory viruses}

Community respiratory viruses including influenza, parainfluenza, adenovirus, HSV, VZV, and RSV cause mild upper respiratory tract infection in patients with normal immunity. They may cause more severe illness along with broncholitis and pneumonia in transplant recipients. ${ }^{[2]}$ Respiratory viral infections increase the risk of superimposed fungal or bacterial pneumonia. ${ }^{[30]}$ HRCT findings of pneumonia due to influenza and respiratory syncytial viruses include ground glass opacity, centrilobular nodules, and tree in bud appearance ${ }^{[31,32]}$ [Figure 3C].

\section{Varicella zoster virus (VZV)}

A majority (upto $90 \%$ ) of varicella zoster pneumoniae occur in immunocompromised patients including renal transplant recipients. Histological features of diffuse alveolar damage are observed in varicella zoster pneumonia. ${ }^{[33]}$ HRCT findings include small less than $10 \mathrm{~mm}$ nodules, nodule with surrounding GGO, diffuse miliary pattern, patchy ground glass opacities, and coalescent nodules ${ }^{[34]}$ [Figure 3D]. These findings usually resolve simultaneously with skin nodules after antiviral treatment; however, they may leave widespread 2-3 $\mathrm{mm}$ residual calcifications. ${ }^{[33]}$

\section{Coronavirus disease 2019 (COVID-19) pneumonia}

The ongoing Coronavirus disease 2019 (COVID-19) pandemic also involves renal transplant patient giving rise to pneumonia. Patient can present with fever, followed by cough, myalgia, chills, and fatigue. The most common chest X-ray and computed tomography abnormality was multifocal patchy opacities. On HRCT, common findings in early disease and mild to moderate cases may be multifocal, bilateral, patchy ground glass opacities [Figure 4]. In later stages and in severe disease, more confluent ground glass opacities, consolidation and an ARDS like picture may be seen. ${ }^{[35]}$ Nasopharyngeal swab real-time reverse transcription polymerase chain reaction (rRT-PCR) for SARS-CoV-2 is essential to make the diagnosis when there is high chance of COVID-19 infection in appropriate clinical setting [Table 6]. ${ }^{[35]}$

\section{Approach to radiological imaging}

The presentation of pulmonary infections in the setting of renal transplant may be either acute or subacute/chronic. An acute process which evolves over hours may be due to bacterial pneumonia. A subacute/chronic process which presents over days to weeks may be due to PCP, viruses, mycobacteria, or fungi.

Imaging patterns can be broadly divided into 1 . Consolidation 2. Nodules 3. Diffuse/interstitial pattern. ${ }^{[36]}$ When the predominant imaging finding is consolidation, the causative organism is commonly tuberculosis or bacteria. The causes of lobar pneumonia include streptococcus and Klebsiella while the causes of bronchopneumonia include staphylococcus and pseudomonas. If nodules are the predominant findings, common etiologies may be fungi, nocardia, tuberculosis, fungi, and viruses. If the size of nodules is less than 10 $\mathrm{mm}$, viral infection is more likely. ${ }^{[2]}$ If the size of nodules is more than $1 \mathrm{~cm}$, then fungal or tubercular infection is more likely. Tree in bud nodules are more common in the setting of tuberculosis as compared to other infections. If cavitation is present in the nodules, then the differentials may include septic emboli or invasive aspergillosis; the latter may show peripheral ground glass halo. Diffuse/interstitial pattern is shown by viruses like CMV and VZV in addition to PCP and miliary tuberculosis. Diffuse pattern may also be caused by bacteria and fungi less frequently.

\section{Conclusion}

To conclude, pulmonary infections are most important infections in renal transplant recipients and the important cause of morbidity and mortality. There is a large spectrum of opportunistic organisms which may cause such infections owing to immunocompromised status. Early diagnosis is essential for specific treatment which is key to recovery. As microbiological diagnosis is often delayed, an empirical therapy is started on the likely diagnosis of infection based on the clinical and radiological patterns. Hence, 
an appropriate approach to these infections becomes mandatory for the favorable outcome of infection.

\section{Financial support and sponsorship}

Nil.

\section{Conflicts of interest}

There are no conflicts of interest.

\section{References}

1. Rosenow EC, Wilson WR, Cockerill FR. Pulmonary disease in the immunocompromised host. 1. Mayo Clin Proc 1985;60:473-87.

2. Wang X, Wang Y, Zhu Q, Guo G, Yuan H. Pulmonary infection in the renal transplant recipients: Analysis of the radiologic manifestations. Radiol Infect Dis 2014;1:3-6.

3. Gulati M, Kaur R, Jha V, Venkataramu NK, Gupta D, Suri S. High-resolution CT in renal transplant patients with suspected pulmonary infections. Acta Radiol 2001;41:237-41.

4. John GT, Shankar V, Abraham AM, Mukundan U, Thomas PP, Jacob CK. Risk factors for post-transplant tuberculosis. Kidney Int 2001;60:1148-53.

5. Singh N, Paterson DL. Mycobacterium tuberculosis infection in solid-organ transplant recipients: Impact and implications for management. Clin Infect Dis 1998;27:1266-77.

6. Unger JD, Rose HD, Unger GF. Gram-negative pneumonia. Radiology 1973;107:283-91.

7. Rozenshtein A, Hao F, Starc MT, Pearson GDN. Radiographic appearance of pulmonary tuberculosis: Dogma disproved. AJR Am J Roentgenol 2015;204:974-8.

8. Sundaram M, Adhikary SD, John GT, Kekre NS. Tuberculosis in renal transplant recipients. Indian J Urol 2008;24:396-400.

9. Pereira M, Gazzoni FF, Marchiori E, Irion K, Moreira J, Giacomelli IL, et al. High-resolution CT findings of pulmonary Mycobacterium tuberculosis infection in renal transplant recipients. Br J Radiol 2016;89:20150686.

10. Leung AN. Pulmonary tuberculosis: The essentials. Radiology 1999;210:307-22.

11. Lal H, Asmita, Mangla L, Prasad R, Gautam M, Nath A. Imaging features of pulmonary infection in post renal transplant recipients: A review. Indian J Transplant 2017;11:13.

12. Chang G-C, Wu C-L, Pan S-H, Yang T-Y, Chin C-S, Yang Y-C, et al. The diagnosis of pneumonia in renal transplant recipients using invasive and noninvasive procedures. Chest 2004;125:541-7.

13. Minero MV, Marín M, Cercenado E, Rabadán PM, Bouza E, Muñoz P. Nocardiosis at the turn of the century. Medicine(Baltimore) 2009;88:250-61.

14. Franquet T, Müller NL, Giménez A, Domingo P, Plaza V, Bordes R. Semiinvasive pulmonary aspergillosis in chronic obstructive pulmonary disease. Am J Roentgenol 2000;174:51-6.

15. Singh N, Husain S, AST infectious diseases community of practice. Invasive aspergillosis in solid organ transplant recipients. Am J Transplant 2009;9 Suppl 4:S180-191.

16. Pinto PS. The CT halo sign. Radiology 2004;230:109-10.

17. Logan PM, Primack SL, Miller RR, Müller NL. Invasive aspergillosis of the airways: Radiographic, CT, and pathologic findings. Radiology 1994;193:383-8.

18. Amr S, Sheikh S, Amir A. Fungal Infection in renal transplant patients. Medical Myocology: Current trends \& future prospects
2015: Taylor \& Francis group, LLC 0.2015. p. 110-146.

19. Song Y, Qiao J, Giovanni G, Liu G, Yang H, Wu J, et al. Mucormycosis in renal transplant recipients: Review of 174 reported cases. BMC Infect Dis 2017;17:283.

20. Franquet T, Müller NL, Giménez A, Martínez S, Madrid M, Domingo P. Infectious pulmonary nodules in immunocompromised patients: Usefulness of computed tomography in predicting their etiology. J Comput Assist Tomogr 2003;27:461-8.

21. Boiselle PM, Tocino I, Hooley RJ, Pumerantz AS, Selwyn PA, Neklesa VP, et al. Chest radiograph interpretation of Pneumocystis carinii pneumonia, bacterial pneumonia, and pulmonary tuberculosis in HIV-positive patients: Accuracy, distinguishing features, and mimics. J Thorac Imaging 1997;12:47-53.

22. Roux A, Gonzalez F, Roux M, Mehrad M, Menotti J, Zahar J-R, et al. Update on pulmonary Pneumocystis jirovecii infection in non-HIV patients. Med Mal Infect 2014;44:185-98.

23. Vogel MN, Brodoefel H, Hierl T, Beck R, Bethge WA, Claussen CD, et al. Differences and similarities of cytomegalovirus and pneumocystis pneumonia in HIV-negative immunocompromised patients thin section CT morphology in the early phase of the disease. Br J Radiol 2007;80:516-23.

24. Bretlau T. Grainger \& Allison's diagnostic radiology, $6^{\text {th }}$ ed. Acta Radiol 2015;56:NP53-NP53.

25. Vogel MN, Vatlach M, Weissgerber P, Goeppert B, Claussen CD, Hetzel J, et al. HRCT-features of Pneumocystis jiroveci pneumonia and their evolution before and after treatment in non-HIV immunocompromised patients. Eur J Radiol 2012;81:1315-20.

26. Kotloff RM, Ahya VN, Crawford SW. Pulmonary complications of solid organ and hematopoietic stem cell transplantation. Am J Respir Crit Care Med 2004;170:22-48.

27. Franquet T, Lee KS, Müller NL. Thin-section CT findings in 32 immunocompromised patients with cytomegalovirus pneumonia who do not have AIDS. Am J Roentgenol 2003;181:1059-63.

28. Horger MS, Pfannenberg C, Einsele H, Beck R, Hebart H, Lengerke $\mathrm{C}$, et al. Cytomegalovirus pneumonia after stem cell transplantation: Correlation of CT findings with clinical outcome in 30 patients. AJR Am J Roentgenol 2006;187:W636-43.

29. Wendt $\mathrm{CH}$. Community respiratory viruses: Organ transplant recipients. Am J Med 1997;102:31-6; discussion 42-3.

30. Ison MG. Respiratory viral infections in transplant recipients. Antivir Ther 2007;12:627-38.

31. Gandhi S, Kute V, Patel K, Sutariya H, Pandya V. Role of high resolution computed tomography of chest in posttransplant pulmonary infection. Indian J Transplant 2017;11:49-54.

32. Ariza-Heredia EJ, Fishman JE, Cleary T, Smith L, Razonable RR, Abbo L. Clinical and radiological features of respiratory syncytial virus in solid organ transplant recipients: A single-center experience. Transpl Infect Dis 2012;14:64-71.

33. Kim EA, Lee KS, Primack SL, Yoon HK, Byun HS, Kim TS, et al. Viral pneumonias in adults: Radiologic and pathologic findings. Radiographics 2002;22(suppl_1):S137-49.

34. Choo HJ, Kim KI, Lee KN. High-resolution CT findings of varicella-zoster pneumonia. J Korean Radiol Soc 2003;49:469-73.

35. Nair V, Jandovitz N, Hirsch JS, Nair G, Abate M, Bhaskaran M, et al. COVID-19 in kidney transplant recipients. Am J Transplant. 2020;20(7):1819-1825. doi: 10.1111/ajt.15967

36. Oh YW, Effmann EL, Godwin JD. Pulmonary infections in immunocompromised hosts: The importance of correlating the conventional radiologic appearance with the clinical setting. Radiology 2000;217:647-56. 